\title{
Microvessel-on-Chip Fabrication for the In Vitro Modeling of Nanomedicine Transport
}

\author{
Sergio Dávila, ${ }^{\dagger}$ Jean Cacheux, ${ }^{\dagger}$ and Isabel Rodríguez*
}

Cite This: ACS Omega 2021, 6, 25109-25115

Read Online

ABSTRACT: Tumor-on-chip devices are becoming ideal platforms to recreate in vitro the particular physiological microenvironment of interest for onco-nanomedicine testing and development. This work presents a strategy to produce a round artificial microvessel on-a-chip device for the study of physiologically relevant nanomedicine transport dynamics. The microchannels have a diameter in the range of the tumor capillaries and a semicircular geometry. This geometry is obtained through an intermediate thermal nanoimprint step using a master mold with square-shaped channel structures produced by standard silicon micromachining or by stereolithography three-dimensional (3D) printing. The working microfluidic chip devices are made by casting polydimethylsiloxane on the imprinted intermediate mold. Artificial blood microvessels are created by seeding human endothelial cells into the round-shaped channels acting as the scaffold. The microchip is connected by $3 \mathrm{D}$ -

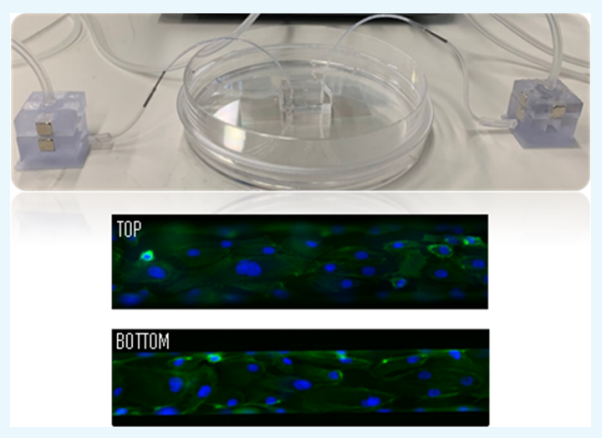
printed reservoirs to a pressure controller, allowing for a fine fluidic control. Under physiological flow conditions, the dynamic interaction of nanoparticles (NPs) with the artificial endothelium was assessed by high-magnification fluorescence microscopy. Overtime, internalization of NPs and clustering was observed and the accumulation rate into the endothelial cells could be characterized in real time.

\section{INTRODUCTION}

Recently, organ-on-chip devices have emerged as preclinical drug-testing platforms with potentially superior predicting power than the conventional two-dimensional (2D) models because these devices are able to recreate in vitro the particular physiological microenvironment and the vascular perfusion scenarios of the target human tissues and organs where the drugs under study are directed to. ${ }^{1-3}$

Organ-on-chip devices are microengineered devices that incorporate the relevant physicochemical parameters and functional characteristics of the organ being modeled and combine physical parameters such as size, pressure, fluid flow, and shear stress, with tissue-specific human cell cultures in a three-dimensional (3D) arrangement. $^{4}$

Particularly, in tumor-on chip devices, the specific features of the tumor microenvironment including the surrounding microvasculature are captured. ${ }^{5,6}$ These devices are especially interesting for evaluating in vitro the complex transport and delivery efficiency of cancer nanomedicines because the major transport barriers involved during their biodistribution can be incorporated and modeled in vitro, on-chip, where real time visualization and monitoring can be carried out.

To date, little is known on nanomedicine transport pathways and on the efficiency of the nanomedicine delivery to the tumor site. ${ }^{7}$ The selective delivery of nanoparticle (NP)-based nanomedicines to solid tumors has been thought to take advantage of the abnormal tumor microvasculature, particularly through the enhanced permeation and retention (EPR) effect. $^{8}$ By the EPR, NPs would extravasate through the interendothelial fenestrations present on the abnormal tumor microvasculature and reach the tumor via diffusion through the leaky vessels. Another possible pathway described for NP extravasation gaining importance is the trans-endothelial pathway, 9,10 whereby the endothelial cells forming the blood vessels actively take part in up-taking NPs from the blood stream, and these NPs trapped in vesiculo-vacuolar cell organelles would be transported through the cytoplasm and eventually would be released across the endothelium into the tumor interstitial matrix. ${ }^{11}$

Thus, the nanomedicine transport pathways are still unclear; yet, the ability to distinguish between passive and active transport pathways across the endothelium is of critical importance for the design of nanomedicines with an effective therapeutic action. ${ }^{10}$ The study of the nanomedicine transport pathways is performed today primarily using animal models, including in vivo imaging methods which involve rather challenging and costly processes. ${ }^{12,13}$

Received: February 9, 2021

Published: September 23, 2021 
a)
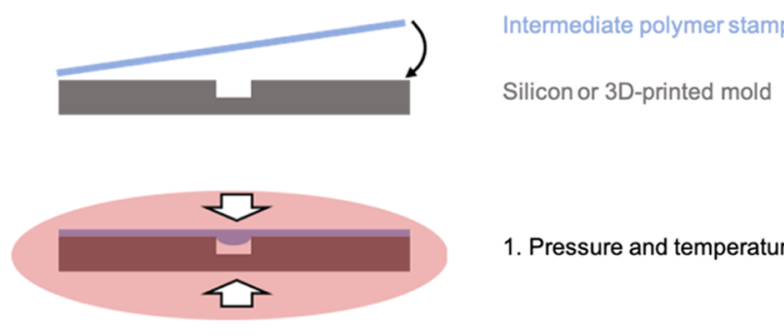

1. Pressure and temperature increase

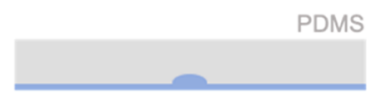

2. PDMS pouring and curing

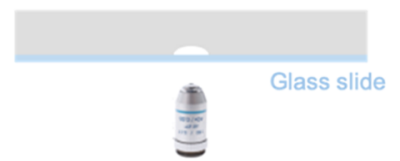

3. Glass slide bonding

b)
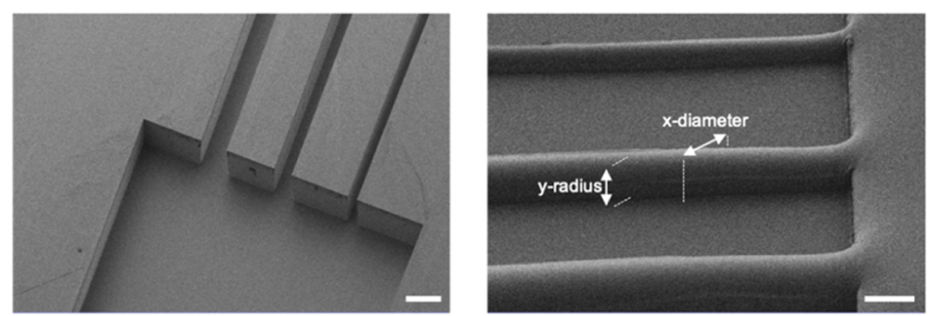

c)

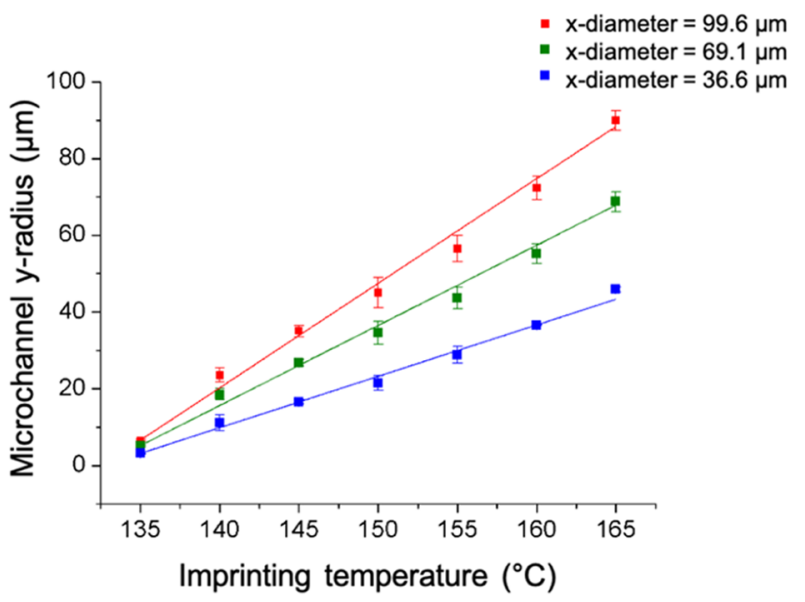

Figure 1. (a) Microvessel microfabrication steps. Initially, a silicon master mold with a rectangular channel is produced via the standard photolithography process. Then, this master mold is used as a mold for thermal imprint onto an IPS to obtain semicircular-shaped channels. On this substrate, PDMS is cast and after demolding the cured slab, it is bonded to a thin glass slide. (b) SEM images of the rectangular silicon master mold (left) and the imprinted IPS with a circular channel (right) used as a working mold. (c) Microchannel $y$-radius vs the imprinting temperature linear dependence for different channel $x$-diameter.

Hence, to shed some light into the NP transport pathways in reaching the solid tumor, artificial vessel-on-a-chip modules including components of the physiological in vivo conditions would be of great practical interest.

Recently, Chen and coworkers have studied the uptake of NPs into endothelial cells lined on microfluidic channels under physiological shear flow conditions. ${ }^{14}$ However, they employ a commercial standard chip which has large channel sizes and does not reproduce the microcapillary dimensions in tumors.

Currently, a few models have been developed for recapitulating the human microvasculature-on-a-chip. These include different approaches depending on the research question to answer.

Generally, in vitro blood vessel models have been produced on hydrogels for studying angiogenesis. ${ }^{15}$

However, these models are not suitable for the highmagnification microscopy imaging of NPs, which will be required to trace the NP transport pathways across an endothelium.

Commonly, microvessel-on-chip devices have been made in polydimethylsiloxane (PDMS) from a master mold fabricated either in silicon or in a polymer; a recent review has summarized these developments. ${ }^{16}$ Because standard photo- 
lithography processes produce typically square profile channels, one of the design issues to address when it comes to construct microvessel-on-a-chip devices is to achieve small (typically smaller than $200 \mu \mathrm{m}$ in diameter) and circular microchannels as more physiological scaffolds to form an artificial confluent endothelial layer. The square profile channels have sharp corners where a nonhomogeneous flow and fluid shear stress distribution at these areas would give rise to a nonhomogeneous convective and diffusive mass transport across the channel thus impacting the NP cellular uptake. In addition, the differences in shear stress across the channel will cause variations on the physiology of the endothelial cell lining. ${ }^{17}$ Furthermore, to produce artificial microvessels, it is required to have a homogeneous and confluent endothelial coverage forming a close endothelial barrier over the channel surface. Previous studies have already shown that the distribution of endothelial cells along the square microchannel is not homogeneous whereby cell adhesion is higher in the middle and scarce at the corners. ${ }^{18}$

During the past years, different approaches have been developed to produce round-shaped channels. Cylindrical microchannels have been created by micromilling on PMMA combined with hot embossing and soft lithography processes. A minimum channel diameter of $200 \mu \mathrm{m}$ was reported. ${ }^{19}$ More frequently, the well-known temperature reflow process of ridges made on thermoplastic materials has been used. ${ }^{17,20,21}$ However, this method offers poor control of the channel cross section. An innovative approach was described by Vecchione $e t$ al., whereby round-shaped channels were produced by spincoating PDMS on microfabricated square trenches; as the PDMS due to surface tension accumulated at the corners, the square channels became rounded. ${ }^{18}$ This approach has been later on employed to create artificial microvessels recapitulating closely the hemodynamics of arteries. ${ }^{22}$ Needles have also been used as a template to form round microvessels into PDMS or hydrogels. ${ }^{23-25}$ Controlled microextrusion of a thixotropic ink has also been employed as a template to cast PDMS channels. ${ }^{26}$ Other approaches to produce a circular channel geometry imply complicated microfabrication processes such as the recently developed backside photolithography $^{27}$ or gray-scale dual-projection lithography, ${ }^{28}$ which are not suitable to produce the large numbers of devices that are required for biological applications. Recently, multidepth round microchannels have been achieved through a process that uses inflated air pressure to deform a partially cured PDMS film. ${ }^{29}$ However, the process has limitations in resolution (about $100 \mu \mathrm{m}$ ) and design layout. Some of the latest approaches have been recently reviewed. ${ }^{30}$

We present hereafter a practical fabrication process to produce small artificial microvessels covered with endothelial cells and with a semicircular geometry which is amenable to high-magnification microscopy characterization. The approach is based on the fabrication of a master mold either in silicon through a photolithographic process or in a polymer by digital light processing-stereolithography (DLP-SLA) 3D printing. By a controlled thermal imprint step, a semicircular shape is obtained from the rectangular master mold. The working devices are produced by soft-lithography replication in PDMS and subsequently sealed against a thin cover glass. Artificial vessels are then created by seeding human umbilical vein endothelial cells (HUVECs) into these channels acting as a scaffold. The microvessels are then connected to a pressure controller via 3D-printed reservoirs to adjust the fluid flow and shear stress. A blood analogue medium is flown into the channels recapitulating closely the tumor microvessel microflow conditions. Transport studies of fluorescent NP and interaction with the vascular endothelium under relevant physiological conditions (in terms of shear flow, viscosity, temperature, and pressure) on this artificial microvessel are then performed in real time.

\section{RESULTS AND DISCUSSION}

Semicircular Microchannel Fabrication Compatible with 3D Printing. A microfabrication process was implemented to produce microvessels with inner diameters of 30 , 60 , and $90 \mu \mathrm{m}$ to be on the size range of tumor capillaries. ${ }^{31}$ The three channels were parallelized closely on the same chip in such a way that the channels could be visualized simultaneously under the microscope.

To allow for the microscopic visualization of the NP interaction with the HUVECs covering the walls of the microchannels using high-magnification objectives, which means short working distance (typically $\sim 150 \mu \mathrm{m}$ ), the channels were made semicircular to be able to place the microscope objective as close as possible to the cells through a thin glass slide, avoiding light scattering effects from round contours.

To make the semicircular channels, a microfabrication procedure involving sequential steps of photolithography, plasma micromachining and nanoimprinting replication was implemented. Initially, the designed channels were patterned onto silicon wafers by maskless photolithography onto a photoresist. The patterned resist served as a selective mask layer for a process of plasma ion etching to form square-shaped microchannel trenches into the silicon. The substrates were used as master molds for a subsequent thermal imprint step onto an intermediate polymer stamp (IPS) material under controlled conditions to convert the channels into semicircular-shaped ridges. This IPS substrate was then used as the working mold for casting PDMS liquid polymer precursors to create the working microchannel substrates (cf. Figure 1a,b).

This fabrication process was also carried out using DLPSLA 3D-printed mold substrates. Due to the lower resolution of the DLP-SLA technique compared to traditional microfabrication, larger channels of the order of $200 \mu \mathrm{m}$ were made. Because the 3D-printed molds are produced by the photopolymerization of a thermoset resin, the produced molds on the cross-linked polymer material were suitable to be used as a master template for thermal imprint replication onto the IPS material.

A key advantage of the process proposed to form semicircular-shaped ridges imprinted onto the IPS is that since the mold channel walls do no enter in contact with the IPS during the thermal imprinting step, very smooth channel surfaces are obtained. This is because IPS, being a material designed with low surface energy, flows into the channel due to the pressure applied, creating a positive convex smooth surface meniscus. Furthermore, this intermediate step allows eliminating the roughness due to the additive layering process during 3D printing. On the other hand, the roughness obtained out of the channel is not an issue as these areas are bonded to a glass slide.

From the experimental thermal imprint results with the silicon mold, it is possible to predict the channel y-radius as a function of the nanoimprint temperature for the three different channel diameters designed for our experiments (cf. Figure 
1c). It is then possible to define the temperature to get a semicircular channel profile (i.e., $y$-radius $=d_{x} / 2$ ).

Similar results were observed with the polymeric $3 \mathrm{D}$-printed mold. Nonetheless, if using alternative materials or different channel sizes, it would be necessary to realize a calibration because other variables such as the material's thermal properties and surface energy influence the nanoimprint polymer filling process. ${ }^{32}$

Endothelial Lining onto Microchannel Scaffolds. Initially, the adhesion and proliferation rate of the HUVECs were assayed in order to adjust the seeding conditions and to determine the time required for the HUVECs to form a confluent endothelium monolayer covering the entire surface of the channels as a function of the initial cell concentration flown into the microchannel.

The cell-adhesive protein treatment for the PDMS channel surface was selected between fibronectin and gelatin on the basis of the HUVEC proliferation rate and morphology on the substrates. Both the fibronectin and gelatin coatings supported HUVEC adhesion and proliferation (Supporting Information, Figure S1). However, the proliferation rate of the HUVECs on the surfaces treated with fibronectin showed the typical growth profile as the control, while the HUVECs seeded on to the PDMS surfaces treated with gelatin showed a somewhat delayed growth. The nontreated PDMS surface did not support growth whatsoever. The maximum growth of the HUVECs was achieved at day 9 where the cells reached confluence and covered the surface completely. At day 10, the cultures reached a stationary growth, followed by a decline phase. Consequently, fibronectin was selected in the subsequent experiments to treat the PDMS channel surfaces before the HUVEC seeding process inside the microchannels.

Figure 2 shows the HUVECs cultured within the microfluidic channels covering the entire lumen where cell confluence reached within $24 \mathrm{~h}$. Figure $2 \mathrm{a}$ shows the HUVEC lining on a microchannel device made from the silicon master, while Figure $2 \mathrm{~b}$ shows the HUVECs seeded into a microchannel fabricated from the 3D-printed master. In both cases, on the top view of the fluorescent images, we can observe that some cells are out of focus at the sides, which is indicative of the rounded curvature at the top part of the channel.

A close-up look at the endothelium morphology covering the microchannel was obtained by scanning electron microscopy (SEM). The images are displayed in Figure 2c whereby the HUVECs are seen forming a compact endothelium layer with tight junctions and the typical flatten morphology with the nucleus protruding lightly. A cell layer of $1 \mu \mathrm{m}$ thickness was estimated from the SEM images.

NP Dynamic Interaction with the Endothelial Microvessel Wall. The interaction of $200 \mathrm{~nm}$ polystyrene NPs with the cellular endothelium formed around the microchannel was studied. The polystyrene NPs were used with a dilution ratio of $1: 5000(\mathrm{v} / \mathrm{v})$ to minimize interparticle hydrodynamic interactions and to ensure single NP tracking during the observation period.

The NPs diluted in the cell media were introduced into the microchannel and flown through with a mean flow velocity ranging from 0.1 to $0.5 \mathrm{~mm} / \mathrm{s}$ by applying a pressure drop between 1 and $20 \mathrm{mbar}$ for $30 \mathrm{~min}$ for 1 , 2, or $4 \mathrm{~h}$ periods. These time steps were chosen because during these periods, the cells did not show any sign of reduced viability.

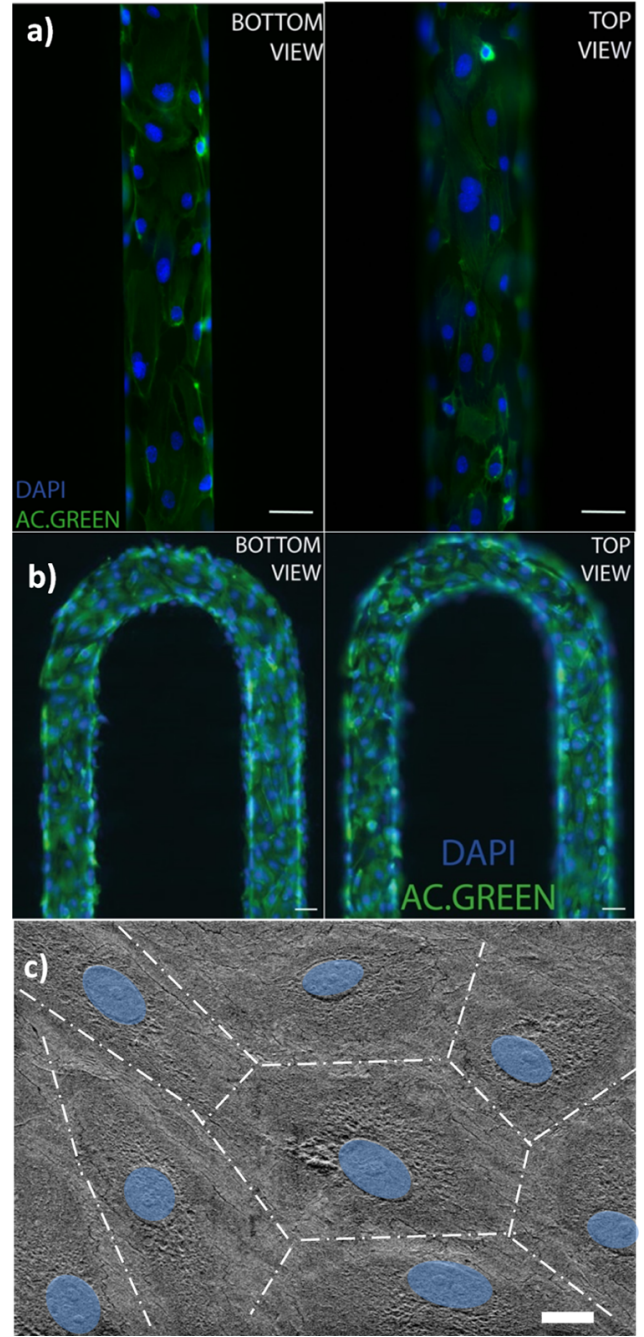

Figure 2. Fluorescence images of the HUVEC adhesion with actin (green) and nucleus (blue) covering the surface of the microchannels obtained from (a) a photolithography master mold and (b) a 3Dprinted master mold (scale bar $=100 \mu \mathrm{m})$. (c) SEM images of HUVECs on a microfluidic device (nucleus colored in blue) (scale bar $=10 \mu \mathrm{m})$.

Figure 3 shows the accumulation of the NP inside the endothelial cell's lining. Figure $3 a-d$ shows the progressive accumulation of NPs inside the cells near the cells' nucleus. A clear accumulation of NPs around the nucleus was seen after 2 $\mathrm{h}$ of interaction (cf. Figure 3c,d).

To be certain that the NPs were indeed inside the cells' cytoplasm and not externally attached, confocal laser scanning microscopy was conducted. The images acquired at several focal planes were stacked ( $z$-stacking) to reconstruct a 3D image of the NP-relative position inside the cells. Figure $3 \mathrm{e}-\mathrm{g}$ shows images where the NPs up taken by the cells are accumulated inside the cytoplasm.

A close-up confocal image inside the cell cytoplasm in Figure $3 \mathrm{~g}$ shows the internalized NP distribution at different heights with an accumulation near the nucleus (white arrows).

\section{CONCLUSIONS}

A simple and predictable microfabrication approach to achieve semicircular-shaped microchannels has been described based on a controlled intermediate thermal nanoimprint step using a 

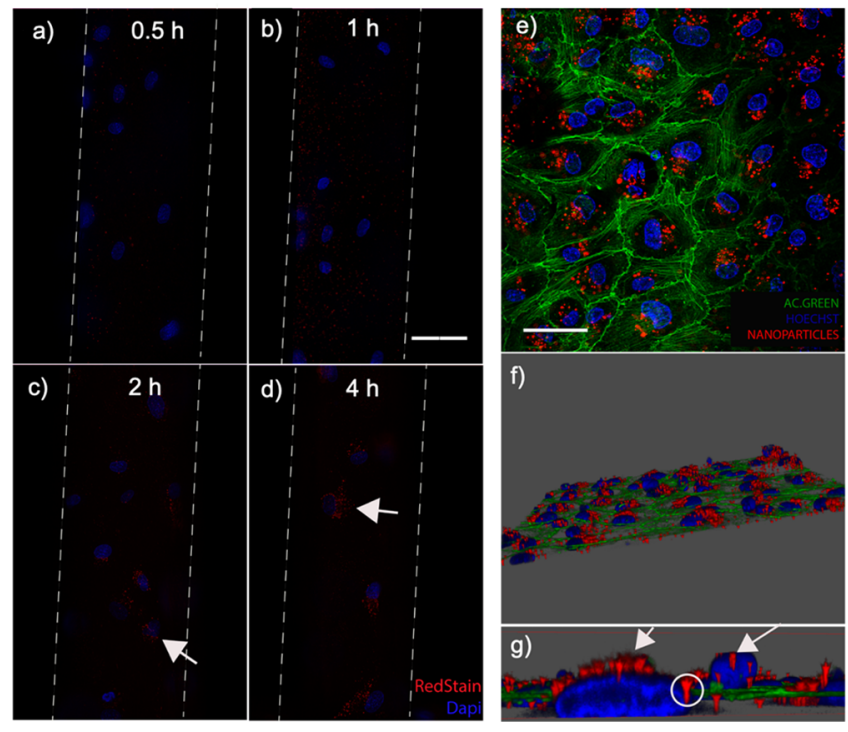

Figure 3. $(a-d)$ Dynamic NP uptake and accumulation into the HUVEC lining of an artificial microvessel for $0.5-4 \mathrm{~h}$ under flow conditions. (e-g) Confocal images of NP internalized by the HUVECs [nucleus (blue), rhodamine-NP (red), and cell actin (green) $]$. Scale bar $=100 \mu \mathrm{m}(\mathrm{a}-\mathrm{d})$. Scale bar $=50 \mu \mathrm{m}(\mathrm{e})$.

master mold fabricated by standard silicon micromachining or by DLP-SLA on thermoset photopolymers. DLP-SLA provides for a quick and practical method for the fast prototyping of microchannels with coarse $X Y$ resolution above $200 \mu \mathrm{m}$. Through the intermediate imprint step, it is possible to eliminate the microchannel side wall roughness inherent to the additive process.

Microchannels are produced by soft lithography using the intermediate-imprinted substrates as working molds and then are sealed onto thin glass cover slides giving rise to enclosed microchannels with a semicircular geometry and allowing for high-magnification optical imaging.

The artificial microvessels have been created by seeding HUVECs onto the microchannels covering the walls and forming a continuous endothelium. Connection through 3Dprinted reservoirs allowed for pumping a shear thinning viscous cell medium at a controlled flow velocity and shear rate in addition to body temperature to mimic physiological vessel conditions.

Assessment of NP uptake and accumulation into the HUVECs under these conditions was carried out and it was seen that NPs were readily up taken by the endothelial cells.

Moving forward, advanced studies are on-going to assess in vitro the trans-vascular NP transport through the vascular endothelium.

\section{EXPERIMENTAL SECTION}

Semicircular Microchannel Fabrication. Silicon Master Mold Micromachining. Initially, silicon wafers were cleaned by $\mathrm{O}_{2}$ plasma (Tepla) for $5 \mathrm{~min}$ at $400 \mathrm{~W}$. To enhance adhesion, the wafers were spin-coated with a primer at 2000 $\mathrm{rpm}$ for $1 \mathrm{~min}$, followed by a $120{ }^{\circ} \mathrm{C}$ baking step for $2 \mathrm{~min}$. Over the primed wafers, a photoresist (AZ1512, Microchemicals) was spin-coated at $1500 \mathrm{rpm}$ for $1 \mathrm{~min}$ and baked at $90{ }^{\circ} \mathrm{C}$ for $1 \mathrm{~min}$. The resist was then exposed to a laser beam writing process (Heidelberg, DWL 66+, $405 \mathrm{~nm}$ ) of the microchannel 2D design. Subsequently, the resist was developed for $1 \mathrm{~min}$ into the appropriate developer (AZ351B, Microchemicals). The resist-patterned wafers were submerged into a buffered HF solution for $60 \mathrm{~s}$ to remove the native silicon oxide before conducting a cryogenic inductively coupled plasma reactive ion etching (ICP-RIE). The process parameters were $19 \mathrm{sccm}$ of $\mathrm{SF}_{6}$ and $5 \mathrm{sccm}$ of $\mathrm{O}_{2}$ at a forward power of $15 \mathrm{~W}$ with inductive coupled plasma at $275 \mathrm{~W}, 30$ mTorr at $-185{ }^{\circ} \mathrm{C}$. These conditions lead to an etching velocity of approx. $5 \mu \mathrm{m} / \mathrm{min}$. After the process, the remaining resist was removed using acetone.

DLP-SLA 3D-Printed Master Mold. This was achieved using an Asiga Max 27X printer on a Dental Model resin (Asiga) at $35{ }^{\circ} \mathrm{C}$ yielding a layer with a $z$-resolution of $25 \mu \mathrm{m}$. The microchannel dimensions were typically $150 \mu \mathrm{m}$ width by $250 \mu \mathrm{m}$ height. The mechanical resistance of the printed mold was better when the channels were printed parallel to the printing direction (Supporting Information, Figure S2). The $3 \mathrm{D}$ printed mold was baked at $70{ }^{\circ} \mathrm{C}$ overnight prior to the thermal imprinting step in order to induce further cross-linking by evaporation of any remaining solvent.

Replication through Thermal Imprint and Chip Fabrication. A commercial polymer film denoted as IPS (TIP1, Obducat) was utilized to copy silicon and 3D-printed molds obtaining a semicircular channel shape. The thermal imprint process was carried out at the given temperature (from 135 to $165{ }^{\circ} \mathrm{C}$ ) and constant pressures of 20 bar for $1 \mathrm{~min}$ and $40 \mathrm{bar}$ for another minute by means of a semiautomated nanoimprint lithography machine (EITRE, Obducat). The IPS was then cooled down to $70{ }^{\circ} \mathrm{C}$ before demolding. Temperature, pressure, and duration of each step were set, thanks to Obducat dedicated software. The circular-shaped ridges created through this process were used as a template to cast PDMS to form the microchannels. (The semicircular profile of the PDMS replicated channels is displayed in Supporting Information, Figure S3.) The outlet and inlet were made using a surgical puncher. Finally, the microchannels were closed by bonding the PDMS-casted channels onto a thin glass cover slide (VWR, $22 \times 50 \mathrm{~mm}$, no. 1) by oxygen plasma activation $\left(50 \mathrm{~mL} / \mathrm{min} \mathrm{O}_{2}, 50 \mathrm{~W}, 30 \mathrm{~s}\right)$.

Cell Culture Materials. Adhesion and Proliferation into the Culture Plate. Primary HUVECs (Sigma) were used and expanded until $80-90 \%$ between passages $(2-11)$ before being used in the experiments. HUVECs were cultured in the endothelial growth medium (EGM) ready-to-use (SigmaAldrich) and incubated at $37{ }^{\circ} \mathrm{C}$ and $5 \% \mathrm{CO}_{2}$, trypsinized with Trypsin- ethylenediaminetetraacetic acid solution (SigmaAldrich), and collected when $80-90 \%$ confluence was reached before seeding the HUVECs at $10^{4}-10^{7}$ cells $/ \mathrm{mL}$ concentration (depending on the experiment). The preparation of the adhesive surfaces was carried out by incubation with $0.1 \%$ gelatin as used in previous study or with $0.05 \%$ human fibronectin solutions.

The HUVEC proliferation was monitored by the resazurin reduction assay. ${ }^{33}$

Cell Culture Experiments in Microfluidic Devices. Initially, the PDMS microchannel walls were functionalized with $0.05 \%$ human fibronectin or $0.1 \%$ gelatin. HUVECs were seeded at a concentration in the range of $1-20$ billion cells $/ \mathrm{mL}$ by flushing the cell suspension into the channel. After the channel was filled, the flow was stopped to allow the cells to attach guided by gravity. Then, the reservoirs were filled with a fresh medium and the chip was placed inside an incubator on a mini table rocker (Supporting Information, Figure S4) in order to renew 
the cell culture medium, while the cells were proliferating covering the entire surface.

Immunostaining. Cells were fixed with $4 \%$ paraformaldehyde (Sigma-Aldrich) at $4{ }^{\circ} \mathrm{C}$, permeabilized with $0.1 \%$ triton X-100 (Sigma-Aldrich) at room temperature, and blocked with bovine serum albumin 2\% (Sigma-Aldrich) for $45 \mathrm{~min}$ at room temperature. Then, cells were incubated with DAPI (Molecular probes) for nucleus labeling and actin-green (molecular probes) for cytoskeleton labeling for $20 \mathrm{~min}$ at room temperature. To visualize the living cells during the NP flow studies, they were labeled with bisBenzimide H 33342 trihydrochloride (Hoechst 33342, Sigma-Aldrich). SEM images (FSEM Auriga, Carl Zeiss) were taken after a thin layer of gold was sputter-coated.

NP Fluidic Operations. Sample Dilution into a Blood Analogue. To mimic the physiological conditions of human blood, an artificial blood medium was formulated by adjusting the viscosity of the EGM with the addition of xanthan gum according to the literature. ${ }^{34} \mathrm{~A}$ concentration of $0.066 \mathrm{~g} / \mathrm{L}$ was used to obtain a viscosity of $4 \times 10^{-3} \mathrm{~Pa}$ s and a shear rate of $200 \mathrm{~s}^{-1}$. Polystyrene NPs (200 nm Nanosphere, Thermofisher) were added into the blood analogue at a typical $(\mathrm{v} / \mathrm{v})$ ratio of 1:5000 to avoid interparticle hydrodynamic interactions ensuring single NP tracking.

Pressure Control. The flow velocity in the microchannels was established through a PC interface by means of a pressure controller (Fluigent, MFCS-EZ) with a precision of $0.006 \%$ of the pressure range. The output pressure of the controller was connected to the headspace of a 3D-printed reservoir inserted into the microfluidic chip inlet with the desired solutions to flow into the chip. ${ }^{35}$ An analytical solution of the flow velocity into semicircular channels has been given by Federspiel and Valenti ${ }^{36}$ as follows

$$
v=\frac{\Delta P \times D^{2}}{169.568 \times \mu \times L}
$$

with $\Delta P$ being the pressure drop along the channel, $r$ the channel radius, $\mu$ the fluid viscosity, and $L$ the channel length. Thus, following this expression, the flow velocity in our channels was adjusted to that of physiological conditions (in the range of $0-1 \mathrm{~mm} / \mathrm{s}$ ) through the application of a pressure drop by a pressure controller. Hence, the pressure drop ranged between 1 and 20 mbar to achieve a mean flow velocity from 0.1 to $0.5 \mathrm{~mm} / \mathrm{s}$ depending on the microchannel dimensions (cf. Supporting Information, Figure S4).

Image Acquisitions. Fluorescence microscopy images were taken on a fluorescence inverted microscope (Leica Dmi8) coupled to a camera (Hamamatsu Flash 4.0 V3). The chip temperature was controlled at $37{ }^{\circ} \mathrm{C}$ by a microscope heating plate (Okolab). Images were acquired using $40 \times$ and $63 \times$ glycerol immersion objectives. Fluorescence signals were acquired by selecting the appropriated filter sets to the fluorescence dye.

Confocal microscopic images were taken using an SP8 advanced light microscopy unit of the CNB-CSIC, Madrid. All the images were acquired and analyzed with a micromanager.

\section{ASSOCIATED CONTENT}

\section{SI Supporting Information}

The Supporting Information is available free of charge at https://pubs.acs.org/doi/10.1021/acsomega.1c00735.
HUVEC adhesion and proliferation assays, 3D-printed mold substrates, replicated semicircular PDMS channels, principle of operation of the mini rocker system for flushing cell media, and flow calibration plot (PDF)

\section{AUTHOR INFORMATION}

\section{Corresponding Author}

Isabel Rodríguez - Madrid Institute for Advanced Studies in Nanoscience (IMDEA Nanoscience), Madrid 28049, Spain; 다이.org/0000-0002-7178-8275; Email: i.rodriguez@ imdea.org

\section{Authors}

Sergio Dávila - Madrid Institute for Advanced Studies in Nanoscience (IMDEA Nanoscience), Madrid 28049, Spain

Jean Cacheux - Madrid Institute for Advanced Studies in Nanoscience (IMDEA Nanoscience), Madrid 28049, Spain

Complete contact information is available at:

https://pubs.acs.org/10.1021/acsomega.1c00735

\section{Author Contributions}

${ }^{\dagger}$ S.D. and J.C. have contributed equally to this work.

\section{Notes}

The authors declare no competing financial interest.

\section{ACKNOWLEDGMENTS}

This work was performed within the framework of the EVONANO project funded by the European Union's Horizon 2020 FET Open programme under grant agreement no. 800983 and partially funded by the "Severo Ochoa" Programme for Centres of Excellence in R\&D (MINECO, Grant SEV-2016-0686). The authors are grateful to M.R. Osorio for his valuable assistance in microfabrication.

\section{REFERENCES}

(1) Esch, E. W.; Bahinski, A.; Huh, D. Organs-on-Chips at the Frontiers of Drug Discovery. Nat. Rev. Drug Discovery 2015, 14, 248260

(2) Mittal, R.; Woo, F. W.; Castro, C. S.; Cohen, M. A.; Karanxha, J.; Mittal, J.; Chhibber, T.; Jhaveri, V. M. Organ-on-chip models: Implications in drug discovery and clinical applications. J. Cell. Physiol. 2019, 234, 8352-8380.

(3) Balijepalli, A.; Sivaramakrishan, V. Organs-on-Chips: Research and Commercial Perspectives. Drug Discovery Today 2017, 22, 397403.

(4) Bhatia, S. N.; Ingber, D. E. Microfluidic Organs-on-Chips. Nat. Biotechnol. 2014, 32, 760-772.

(5) Sontheimer-Phelps, A.; Hassell, B. A.; Ingber, D. E. Modelling Cancer in Microfluidic Human Organs-on-Chips. Nat. Rev. Cancer 2019, 19, 65-81.

(6) Carvalho, M. R.; Barata, D.; Teixeira, L. M.; Giselbrecht, S.; Reis, R. L.; Oliveira, J. M.; Truckenmüller, R.; Habibovic, P. Colorectal Tumor-on-a-Chip System: A 3D Tool for Precision Onco-Nanomedicine. Sci. Adv. 2019, 5, No. eaaw1317.

(7) Moghimi, S. M.; Simberg, D. Nanoparticle Transport Pathways into Tumors. J. Nanoparticle Res. 2018, 20, 169.

(8) Iyer, A. K.; Khaled, G.; Fang, J.; Maeda, H. Exploiting the Enhanced Permeability and Retention Effect for Tumor Targeting. Drug Discovery Today 2006, 11, 812-818.

(9) Pandit, S.; Dutta, D.; Nie, S. Active transcytosis and new opportunities for cancer nanomedicine. Nat. Mater. 2020, 19, 478480.

(10) Skotland, T.; Sandvig, K. Transport of nanoparticles across the endothelial cell layer. Nano Today 2021, 36, 101029. 
(11) Sindhwani, S.; Syed, A. M.; Ngai, J.; Kingston, B. R.; Maiorino, L.; Rothschild, J.; MacMillan, P.; Zhang, Y.; Rajesh, N. U.; Hoang, T.; Wu, J. L. Y.; Wilhelm, S.; Zilman, A.; Gadde, S.; Sulaiman, A.; Ouyang, B.; Lin, Z.; Wang, L.; Egeblad, M.; Chan, W. C. W. The Entry of Nanoparticles into Solid Tumours. Nat. Mater. 2020, 19, $566-575$.

(12) Man, F.; Lammers, T.; de Rosales, R. T. Imaging nanomedicine-based drug delivery: a review of clinical studies. Mol. Imaging Biol. 2018, 20, 683-695.

(13) Lu, L.; Sun, Y.; Wan, C.; Hu, Y.; Lo, P.-C.; Lovell, J. F.; Yang, $\mathrm{K}$.; Jin, H. Role of intravital imaging in nanomedicine-assisted anticancer therapy. Curr. Opin. Biotechnol. 2021, 69, 153-161.

(14) Chen, Y. Y.; Syed, A. M.; MacMillan, P.; Rocheleau, J. V.; Chan, W. C. W. Flow Rate Affects Nanoparticle Uptake into Endothelial Cells. Adv. Mater. 2020, 32, 1906274.

(15) Kwak, T. J.; Lee, E. In vitro modeling of solid tumor interactions with perfused blood vessels. Sci. Rep. 2020, 10, 20142.

(16) Luque-González, M. A.; Reis, R. L.; Kundu, S. C.; Caballero, D. Human Microcirculation-on-Chip Models in Cancer Research: Key Integration of Lymphatic and Blood Vasculatures. Adv. Biosyst. 2020, 4, 2000045.

(17) Li, X.; Mearns, S. M.; Martins-Green, M.; Liu, Y. Procedure for the Development of Multi-Depth Circular Cross-Sectional Endothelialized Microchannels-on-a-Chip. J. Vis. Exp. 2013, 80, e50771.

(18) Vecchione, R.; Pitingolo, G.; Guarnieri, D.; Falanga, A. P.; Netti, P. A. From square to circular polymeric microchannels by spin coating technology: a low cost platform for endothelial cell culture. Biofabrication 2016, 8, 025005.

(19) Jang, M.; Kwon, Y. J.; Lee, N. Y. Non-photolithographic plasticmold-based fabrication of cylindrical and multi-tiered poly(dimethylsiloxane) microchannels for biomimetic lab-on-a-chip applications. RSC Adv. 2015, 5, 100905-100911.

(20) Choi, J. S.; Piao, Y.; Seo, T. S. Fabrication of a Circular PDMS Microchannel for Constructing a Three-Dimensional Endothelial Cell Layer. Bioprocess Biosyst. Eng. 2013, 36, 1871-1878.

(21) Rho, H. S.; Veltkamp, H.-W.; Baptista, D.; Gardeniers, H.; Le Gac, S.; Habibović, P. A 3D Polydimethylsiloxane MicrohourglassShaped Channel Array Made by Reflowing Photoresist Structures for Engineering a Blood Capillary Network. Methods 2020, 190, 63-71.

(22) Cho, M.; Park, J.-K. Fabrication of a Perfusable 3D In Vitro Artery-Mimicking Multichannel System for Artery Disease Models. ACS Biomater. Sci. Eng. 2020, 6, 5326-5336.

(23) Sfriso, R.; Zhang, S.; Bichsel, C. A.; Steck, O.; Despont, A.; Guenat, O. T.; Rieben, R. 3D Artificial Round Section Micro-Vessels to Investigate Endothelial Cells under Physiological Flow Conditions. Sci. Rep. 2018, 8, 1-13.

(24) Kwak, T. J.; Lee, E. In vitro modeling of solid tumor interactions with perfused blood vessels. Sci. Rep. 2020, 10, 1-9.

(25) Tan, A.; Fujisawa, K.; Yukawa, Y.; Matsunaga, Y. T. Bottom-up fabrication of artery-mimicking tubular co-cultures in collagen-based microchannel scaffolds. Biomater. Sci. 2016, 4, 1503-1514.

(26) Xing, J.; Rong, W.; Sun, D.; Wang, L.; Sun, L. Rapid fabrication of rounded microchannels via extrusion printing of molds using a thixotropic ink. Sens. Actuators, B 2017, 248, 613-621.

(27) Fenech, M.; Girod, V.; Claveria, V.; Meance, S.; Abkarian, M.; Charlot, B. Microfluidic Blood Vasculature Replicas Using Backside Lithography. Lab Chip 2019, 19, 2096-2106.

(28) He, R.; Yunus, D.; Uhl, C.; Shi, W.; Sohrabi, S.; Liu, Y. Fabrication of Circular Microfluidic Channels through Grayscale Dual-Projection Lithography. Microfluid. Nanofluidics 2017, 21, 13.

(29) Nguyen, T. Q.; Park, W.-T. Fabrication Method of Multi-Depth Circular Microchannels for Investigating Arterial Thrombosis-on-aChip. Sens. Actuators, B 2020, 321, 128590.

(30) Fleischer, S.; Tavakol, D. N.; Vunjak-Novakovic, G. From arteries to capillaries: approaches to engineering human vasculature. Adv. Funct. Mater. 2020, 30, 1910811.

(31) Caballero, D.; Blackburn, S. M.; de Pablo, M.; Samitier, J.; Albertazzi, L. Tumour-Vessel-on-a-Chip Models for Drug Delivery. Lab Chip 2017, 17, 3760-3771.
(32) Rodriguez, I.; Hernández, J. J. Soft Thermal Nanoimprint and Hybrid Processes to Produce Complex Structures. Nanofabrication: Nanolithography Techniques and their Applications; IOP Publishing, 2020.

(33) Riss, T. L.; Moravec, R. A.; Niles, A. L.; Benink, H. A.; Worzella, T. J.; Minor, L. Cell Viability Assays in Assay Guidance Manual; Sittampalam, G. S., Ed.; Eli Lilly \& Company and the National Center for Advancing Translational Sciences: Bethesda (MD, 2004. https://www.ncbi.nlm.nih.gov/books/NBK144065/.

(34) van den Broek, C. N.; Pullens, R. A. A.; Frøbert, O.; Rutten, M. C. M.; den Hartog, W. F.; van de Vosse, F. N. Medium with BloodAnalog Mechanical Properties for Cardiovascular Tissue Culturing. Biorheology 2008, 45, 651-661.

(35) Alcaide, D.; Cacheux, J.; Dávila, S.; Rodriguez, I. Interfacing Microfluidic Chips: Dual Connector-Reservoir Fixture; Chips and Tips, 2021.

(36) Federspiel, W. J.; Valenti, I. On Laminar Flow in Microfabricated Channels with Partial Semi-Circular Profiles. Open J. Appl. Sci. 2012, 2, 28-34. 